\title{
ZNF750 inhibits the proliferation and invasion of melanoma cells through modulating the Wnt $/ \beta$-catenin signaling pathway
}

\author{
Yong Du', Guozhong LV', Changrui Jing ${ }^{2}$, Junjie Liu ${ }^{3}$, Jing Liu ${ }^{2}$ \\ ${ }^{1}$ Department of Burns and Plastic Surgery, Affiliated Hospital of Jiangnan University, Wuxi, China \\ ${ }^{2}$ Department of Plastic Surgery, Nanjing Medical University Affiliated Wuxi Second Hospital, \\ Wuxi, China \\ ${ }^{3}$ Department of Surgery Yi Xing Guan Lin Hospital, Wuxi, China
}

\begin{abstract}
Introduction. The abnormal expression of Zinc Finger Protein 750 (ZNF750) has been reported in neoplastic diseases. This study investigated the functional role of ZNF750 in the progression of melanoma.

Material and methods. Quantitative real-time PCR and immunohistochemistry (IHC) were performed to detect the expression levels of ZNF750 in patients diagnosed with primary cutaneous malignant melanoma. The correlation between clinical-pathological features and ZNF750 expression were clarified. Cell Counting Kit-8 (CCK-8), colony formation and transwell assays were used to explore the effects of ZNF750 on the proliferation, colony formation, migration and invasion of melanoma cells. Western blot assay was used to evaluate the effects of ZNF750 on regulating epithelial-mesenchymal transition (EMT) related proteins.

Results. ZNF750 expression was down-regulated in human melanoma tissues and cells, and correlated with the clinical-pathological features including tumor size, lymph node metastasis, and Clark classification in patients with melanoma. In addition, overexpression of ZNF750 decreased the proliferation, invasion and suppressed EMT of melanoma cells, whereas ZNF750 depletion showed the opposite effects. Importantly, mechanistic analyses implied that upregulation of ZNF750 inhibited the expression of $\beta$-catenin and the downstream targets (cyclin D1, c-Myc, Bcl-2, MMP2 and MMP9), indicating it could block the activation of Wnt/ $\beta$-catenin pathway. Consistently, knockdown of ZNF750 led to the opposite results.

Conclusions. Together, ZNF750 serves as a tumor suppressor for the development and progression of melanoma through regulating the Wnt $/ \beta$-catenin pathway. This study confirms the involvement of ZNF750 in melanoma progression and may provide a promising therapeutic target for the treatment of melanoma. (Folia Histochemica et Cytobiologica 2020, Vol. 58, No. 4, 255-263)
\end{abstract}

Key words: melanoma; zinc finger protein 750; expression; A375 cells; A2058 cells; proliferation; invasion; EMT markers

\section{Introduction}

Melanoma is a highly malignant tumor that originates from ectodermal neural crest melanocytes, accounting for about $1.5 \%$ of all malignancies [1]. Despite advances in surgical techniques and targeted

Correspondence address: Jing Liu,

NO.68 Zhongshan Road, Liangxi District,

Wuxi, Jiangsu, P.R. China, 214000, China

e-mail: Jing2535@126.com chemotherapies in early-stage melanoma, the 5-year overall survival rate remains unsatisfactory since most patients are diagnosed at advanced stages with local invasion and distal metastases [2-4]. However, the potential molecular mechanisms of melanoma progression remain unclear. Therefore, a better understanding of the occurrence and progression of melanoma is essential for exploring new diagnostic markers and effective treatments.

Zinc Finger Protein 750 gene (ZNF750, Gene Bank Accession No. NM_ 024702) is located at 
$17 \mathrm{q} 25.3$ and encodes 723 amino acid protein, a member of the ZNF family, which contains the typical $\mathrm{C} 2 \mathrm{H} 2$ zinc finger domain [5]. ZNF750 protein is usually expressed in stratified squamous epithelial cells, and is an important regulator of keratinocyte differentiation [6]. ZNF750 is closely related to the incidence and prognosis of a variety of tumors, including squamous cell carcinoma [4] and nasopharyngeal carcinoma [7]. Moreover, ZNF750 can regulate multiple biological phenotypes of cancer cells, including proliferation, migration, invasion, angiogenesis and chemoresistance [8-11]. Liu et al. have used the genome-wide microarray technology based on five microarray datasets on melanoma published between 2000 and 2011, and found the abnormal expression of approximately 200 genes including ZNF750 [12]. Consistently, by using online GEO2R analysis of GSE7553 dataset, we showed in the present paper that ZNF750 was significantly down-regulated in melanoma tissues $(\log \mathrm{FC}=-5.76, \mathrm{p}=0.017)$. These analyses suggest that ZNF750 may have a regulatory effect(s) in melanoma. However, the possible role and mechanisms of ZNF750 in melanoma progression remain unclear.

This study aimed to investigate the effect of ZNF750 on cell growth, migration, invasion in melanoma cells and reveal the underlying regulatory mechanisms.

\section{Materials and methods}

Human specimen. Fifty melanoma tissues from skin of patients ( 45 specimens from the limbs, 3 from the face and 2 from the chest) and paired nonmalignant normal control skin tissues (areas 10-15 cm far from the melanoma tissues) were collected. Patients were diagnosed with primary cutaneous malignant melanoma and had not undergone surgical resection, preoperative radiotherapy or chemotherapy. These patients were collected from 2017 to 2019 at the Drum Tower Clinical College of Nanjing Medical University, Wuxi, China. The study was performed in accordance with the guidelines of the Declaration of Helsinki and the ethical guidelines for medical and health research involving human subjects as established by the National Institutes of Health (NIH) and approved by the ethics committee at the Drum Tower Clinical College of Nanjing Medical University (Jiangsu Province, China). Relevant clinical information was collected and informed consent forms were signed by all enrolled patients.

Gene expression profile analysis. Gene expression chips of melanoma (GSE7553, 82 melanoma tumor tissues and 4 normal skin tissues) were obtained from the Gene Expression Omnibus database (GEO, http://www.ncbi.nlm.nih.gov/ geo). The online software GEO2R (http://www.ncbi.nlm.
nih.gov/geo/geo2R/) was employed to screen differentially expressed genes (DEGs) with the criterion of $|\operatorname{LogFC}|$ $>2$ and adjusted $\mathrm{P}$-value $<0.05$.

Cell culture and transfection. Human low metastatic melanoma (A375, G361 cell lines), and highly metastatic melanoma (A2058, SK-MEL-28 cell lines) [13] and normal human epidermal melanocytes (NHEM cells) were obtained from the American Type Culture Collection (Manassas, VA, USA). All cell lines were maintained in Dulbecco's modified Eagle medium (DMEM, Life Technologies, Carlsbad, CA, USA) supplemented with $10 \%$ fetal bovine serum (FBS, Life Technologies), $100 \mu \mathrm{g} / \mathrm{mL}$ penicillin/streptomycin at $37^{\circ} \mathrm{C}$ with $5 \% \mathrm{CO}_{2}$. The full-length ZNF750 gene was amplified by polymerase chain reaction (PCR) using Phusion Flash High Fidelity PCR Master Mix (Thermo Fisher Scientific, Inc., Waltham, MA, USA). Then, ZNF750 was subcloned into pcDNA3.1 Vector (Invitrogen, Carlsbad, CA, USA), named as ZNF750 group, and empty vector pcDNA3.1 was used as control. Small hairpin RNAs (shRNAs) targeting ZNF750 were synthesized by GenePharma (Shanghai, China), termed as shZNF750. ShRNA-negative control (NC) was used as a scrambled shNC group. Transfections of the vectors or shRNA in melanoma cells were performed by Lipofectamine 2000 (Invitrogen).

Quantitative real-time polymerase chain reaction (qRT-PCR). Total RNA from tissues or cultured cells was extracted with TRIzol reagent (Invitrogen) and reverse transcribed to cDNA using the PrimeScript RT Reagent Kit (TaKaRa Biotech, Dalian, China). PCR assays was conducted by ABI 7500 real-time PCR system (Applied Biosystems, Foster City, CA, USA) using the SYBR Premix Ex Taq GC kit (TaKaRa). All samples were normalized to GAPDH and calculated by $2^{-\Delta \Delta C t}$ method. Primers used were listed as follows: ZNF750: Forward, 5'-GAACAGGTACTGCTTCCTGAGC-3', Reverse, 5'-GAGAGCCTCCGTCATCTGG-3 ; GAPDH: Forward, 5'-CACCCACTCCTCCACCTTTG-3', Reverse, 5'-CCACCACCCTGTTGCTGTAG-3'.

Immunohistochemistry. Briefly, tissues were fixed in $4 \%$ paraformaldehyde and embedded in paraffin. Sections were cut into $4 \mu \mathrm{m}$ slices and stained with primary antibody targeting ZNF750 (1:50, ab121685, Abcam, Cambridge, MA, USA) at $4^{\circ} \mathrm{C}$ overnight. After washing, the sections were incubated with HRP-conjugated goat anti-rabbit IgG secondary antibody for $1 \mathrm{~h}$ at room temperature, incubated with 3, 3'-diaminobenzidine tetrahydrochloride (DAB) as chromogen substrate and finally counterstained with hematoxylin.

Western blot. Proteins were extracted from cells by RIPA lysis buffer (Beyotime, Shanghai, China), and quantified using Bicinchoninic Acid (BCA) protein quantification kit 
(Thermo Fisher Scientific, Inc.) and separated by sodium dodecyl sulfate polyacrylamide gel electrophoresis (SDSPAGE). Proteins were transferred to polyvinylidene fluoride (PVDF) membranes (EMD Millipore, Billerica, MA, USA) and blocked with $5 \%$ nonfat milk for $1 \mathrm{~h}$. The membrane was then incubated with primary antibodies: anti-ZNF750 (1:1000, \#PA5-40743, Thermo Fisher Scientific, Inc.), anti-E-cadherin (1:500, \#ab15148, Abcam), anti-N-cadherin (1:1000, \#ab76057, Abcam), anti- $\beta$-catenin (1:5000, \#ab32572, Abcam), anti-MMP2 (1:1000, \#ab97779, Abcam), anti-MMP9 (1:1000, \#ab38898, Abcam), anti-cyclin D1 (1:200, \#ab16663, Abcam), anti-c-Myc (1:1000, ab32072, Abcam), anti-GAPDH (1:10000, \#ab181602, Abcam). Then, the membranes were immersed into buffer with corresponding horseradish peroxidase-conjugated secondary antibodies (Abcam), visualized by ECL kit (Invitrogen) and detected by an imaging system (Bio-Rad, Hercules, CA, USA).

Cell counting kit-8 $(\mathbf{C C K}-8)$ assay. Briefly, $4 \times 10^{3}$ cells were implanted into each well of $96-w e l l$ plates. Then cells of each well were supplied with $10 \mu \mathrm{L}$ CCK-8 solutions (Dojindo Laboratories, Kumamoto, Japan) and incubated for $2 \mathrm{~h}$. The absorbance was measured at $450 \mathrm{~nm}$ using a Microplate Autoreader (Thermo Fisher Scientific, Inc.).

Colony formation assay. $1 \times 10^{3}$ cells/well were seeded into a 6-well plate and cultured for about two weeks. Then, the cells were fixed by paraformaldehyde (PFA, Sigma-Aldrich, St. Louis, MO, USA) and stained with $0.5 \%$ crystal violet (Solarbio, Beijing, China). Colony numbers were counted and photographed using a microscope (Olympus Ltd., Tokyo, Japan).

Transwell assay. Cell migration and invasive ability was examined using a 24-well transwell plate with $8 \mathrm{~mm}$ pore polycarbonate membrane inserts (Corning Inc., Corning, NY, USA). For the migration assay, $3 \times 10^{4}$ cells/well were seeded in $500 \mu \mathrm{L}$ of serum-free medium and placed in the upper chamber, and $400 \mu \mathrm{L}$ of DMEM medium with $10 \%$ FBS were added in the lower chamber. After maintaining for $36 \mathrm{~h}$, cells in the lower chamber were fixed by $4 \%$ PFA, stained with $0.5 \%$ crystal violet and counted under a microscope. For the invasion assay, the polycarbonate membranes of the upper compartment of the chambers were pre-coated with $100 \mu \mathrm{g}$ Matrigel (BD Biosciences, Mansfield, MA, USA).

Statistical analysis. All data were analyzed by GraphPad Prism 6 (version 3.02, Graph-Pad Software, San Diego, CA, USA) and displayed as mean \pm standard deviation (SD) from at least three independent experiments. Associations between ZNF750 expression and clinicopathological factors were evaluated by Chi-square test. Comparisons between two or more groups were analyzed by student's t-test or one-way ANOVA. ${ }^{*} p<0.05,{ }^{* *} p<0.01$ and ${ }^{* * *} p<0.001$ was regarded statistically significant.

\section{Results}

\section{ZNF750 expression was decreased in melanoma tissues and cells}

The microarray data of GSE7553 were downloaded from GEO database and the differentially expressed genes between melanoma tumor tissue and normal skin tissue samples were analyzed by the online software GEO2R tool. Among the dysregulated genes, ZNF750 exhibited significantly larger relative fold change $(\log \mathrm{FC}=-5.76, \mathrm{p}=0.017$, Fig. 1A) and was downregulated in melanoma tumor tissues, which were selected for further analysis. To verify this finding, the expression of ZNF750 in 50 paired melanoma tissues and corresponding adjacent noncancerous tissues were detected by qRT-PCR and IHC assays. Similarly, ZNF750 was downregulated in melanoma tissues as compared to normal tissues (Fig. 1B). Representative images of IHC staining further confirmed the downregulation of ZNF750 in melanoma tissues (Fig. 1C).

\section{ZNF750 expression and clinicopathological features of melanoma patients}

Based on the median expression value of ZNF750 from qRT-PCR results, the patients were divided into high ZNF750 expression group $(\mathrm{n}=25)$ and low ZNF750 expression group $(n=25)$. The overall survival rate of high ZNF750 expression was higher than that of low ZNF750 expression, indicating that low ZNF750 expression predicted poor prognosis (Fig. 1D). As displayed in Table 1, the expression of ZNF750 was significantly related to tumor size, lymph node metastasis, and Clark classification $(\mathrm{p}<0.05)$.

\section{ZNF750 expression in melanoma cell lines}

The ZNF750 levels were also detected in melanoma cell lines (A375, C8161, A2058 and SK-MEL-1 cells) and normal human epidermal melanocytes (NHEM cells). Decreased ZNF750 mRNA level was observed in melanoma cells compared to NHEM cells (Fig. 1D). Consistently, the protein level of ZNF750 was lower in melanoma cells compared to NHEM cells (Fig. 1E). In particular, A2058 cells had the highest expression of ZNF750, while A375 cells had the lowest expression of ZNF750, which were respectively used for the knockdown and overexpression experiments. These results suggested a critical role of ZNF750 in melanoma progression. 


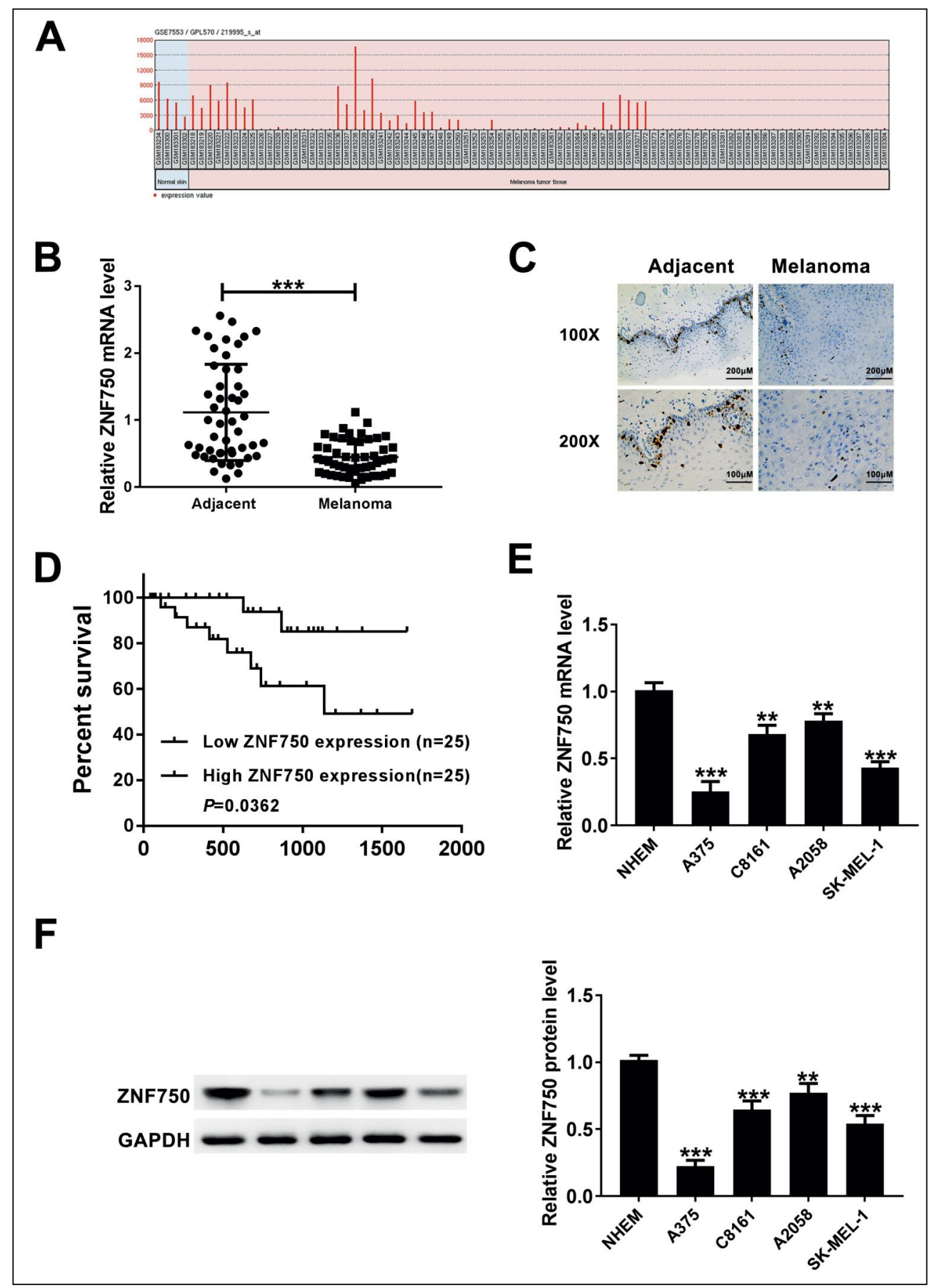

Figure 1. ZNF750 expression was decreased in melanoma tissues and cells. A. ZNF750 expression level in GSE7553 datasets was analyzed by GEO2R. B. ZNF750 expression level in melanoma tissues and corresponding adjacent noncancerous tissues via qRT-PCR. C. IHC assay detected downregulated ZNF750 expression level in melanoma tissues and corresponding adjacent noncancerous tissues (Scale bars $=200 \mu \mathrm{m}$ and $100 \mu \mathrm{m}$ ). D. The overall survival rate of patients with melanoma. qRT-PCR (E) and Western blot (F) assays were used to evaluate the ZNF750 expression in melanoma cell lines (A375, C8161, A2058 and SK-MEL-1 cells) and normal human epidermal melanocytes (NHEM) cells. Results are presented as mean \pm SD. ${ }^{* *} \mathrm{p}<0.01,{ }^{* * *} \mathrm{p}<0.001$.

\section{ZNF750 inhibits the proliferation of melanoma cells}

To further examine the potential role of ZNF750 in the progression of melanoma, ZNF750 was ZNF750 subcloned into pcDNA3.1 vector and overexpressed in A375 cells and knocked down by shRNAs in A2058 cells, and the transfection efficiency was verified by qRT-PCR and western blot assays (Figs. 2A-B). 
Table 1. The relationship between ZNF750 mRNA expression and clinicopathological factors in melanoma tissues

\begin{tabular}{|c|c|c|c|c|}
\hline \multirow[t]{2}{*}{ Characteristics } & \multirow[t]{2}{*}{ Number of patients (\%) } & \multicolumn{2}{|c|}{ ZNF750 mRNA expression } & \multirow[t]{2}{*}{$P$ value } \\
\hline & & Low (< median) & High ( $\geq$ median) & \\
\hline Total number of patients & 50 & 25 & 25 & \\
\hline \multicolumn{5}{|l|}{ Age (years) } \\
\hline$<60$ & $27(54)$ & 14 & 13 & \multirow{2}{*}{0.777} \\
\hline$\geq 60$ & $23(46)$ & 11 & 12 & \\
\hline \multicolumn{5}{|l|}{ Gender } \\
\hline Male & $23(46)$ & 14 & 9 & \multirow{2}{*}{0.156} \\
\hline Female & $27(54)$ & 11 & 16 & \\
\hline \multicolumn{5}{|l|}{ Tumor size } \\
\hline$<2 \mathrm{~cm}$ & $22(44)$ & 7 & 15 & \multirow{2}{*}{$0.047^{*}$} \\
\hline$\geq 2 \mathrm{~cm}$ & $28(56)$ & 18 & 10 & \\
\hline \multicolumn{5}{|l|}{ Lymph node metastasis } \\
\hline Absent & $24(48)$ & 7 & 17 & \multirow{2}{*}{$0.005^{*}$} \\
\hline Present & $26(52)$ & 18 & 8 & \\
\hline \multicolumn{5}{|l|}{ Clark classification } \\
\hline I-II & $21(42)$ & 6 & 15 & \multirow{2}{*}{$0.010^{*}$} \\
\hline III-IV & $29(58)$ & 19 & 10 & \\
\hline
\end{tabular}

*Indicated statistical significance $(\mathrm{p}<0.05)$

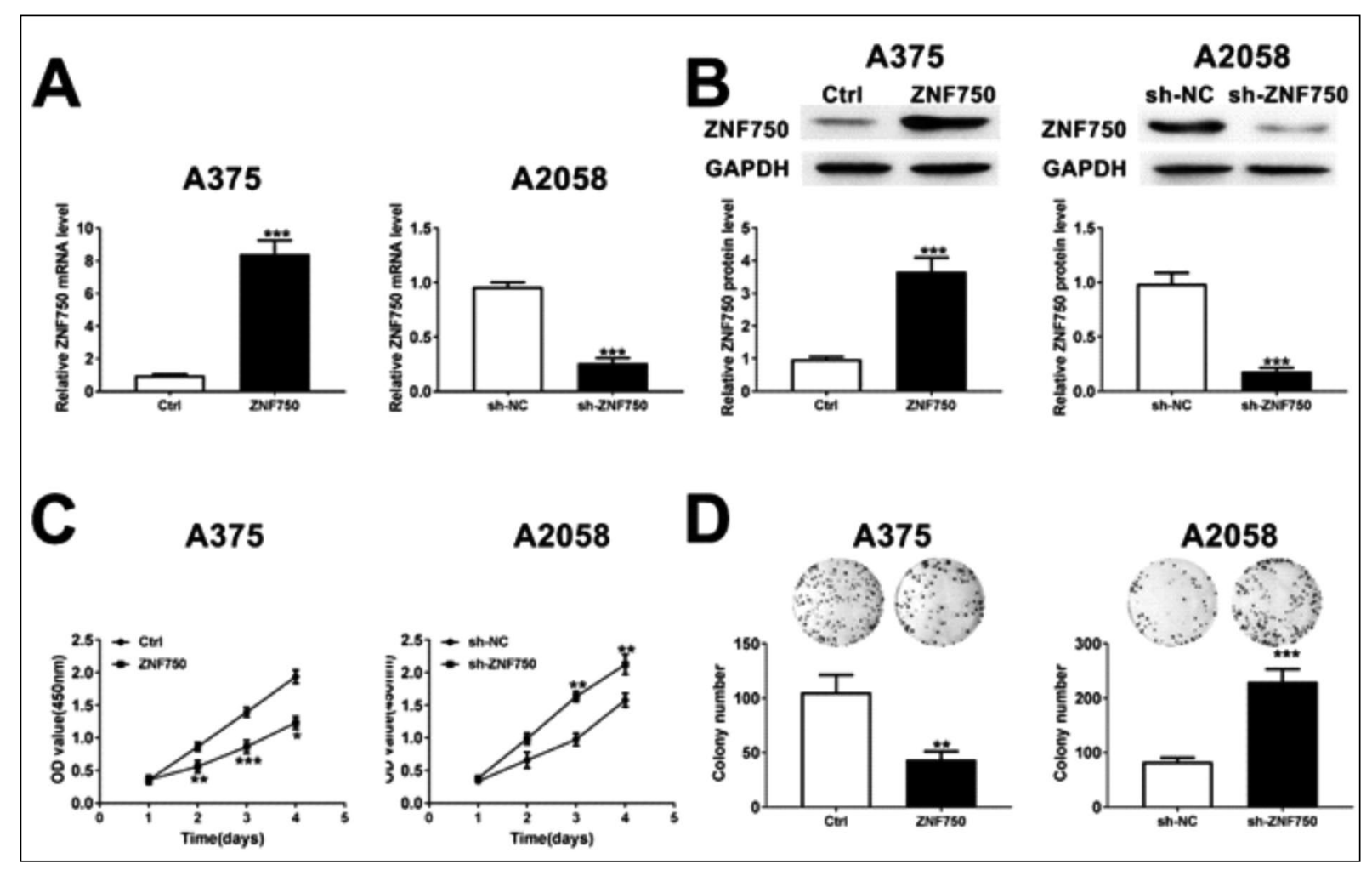

Figure 2. ZNF750 inhibits proliferation of melanoma cells. A-B. The transfection efficiencies of ZNF750 in A375 and A2058 cells were identified by qRT-PCR (A) and western blot (B) analysis. C-D. CCK-8 (C) and colony formation (D) assays were conducted to evaluate the proliferative ability in A375 and A2058 cells. Results are presented as mean \pm SD. ${ }^{*} \mathrm{p}<0.05$, ${ }^{* *} \mathrm{p}<0.01,{ }^{* * *} \mathrm{p}<0.001$. 


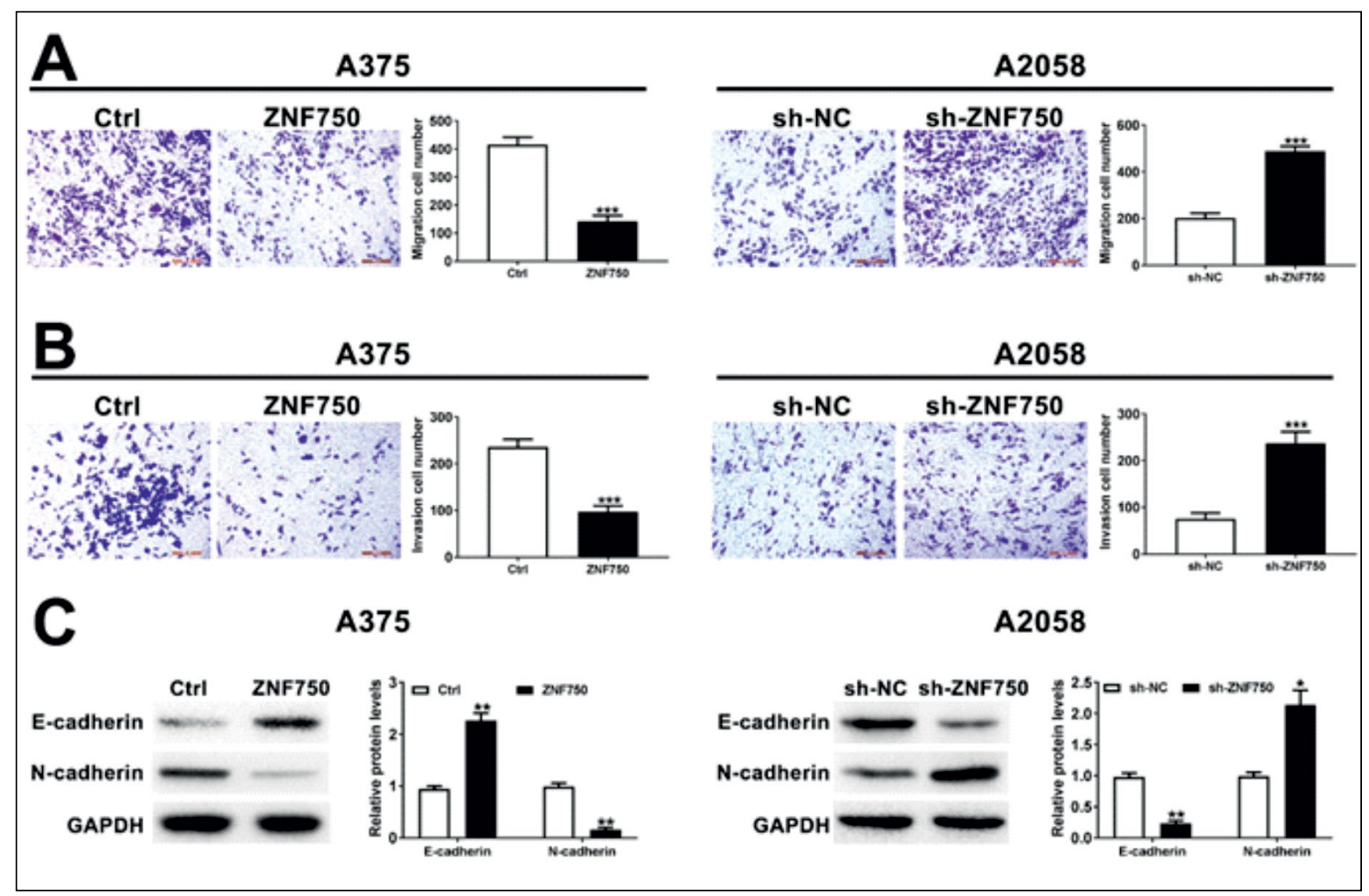

Figure 3. ZNF750 resulted in the inhibition of the migration, invasion and EMT in melanoma cells. A-B. Transwell assay was performed to detect cell migration (A) and invasion (B) in A375 and A2058 cells. B. Western blot assay was used to evaluate the expression of E-cadherin and N-cadherin in A375 and A2058 cells. Results are presented as mean \pm SD. ${ }^{*} \mathrm{p}<0.05,{ }^{* *} \mathrm{p}<0.01,{ }^{* *} \mathrm{p}<0.001$.

CCK-8 and colony formation assays indicated that overexpression of ZNF750 significantly inhibited proliferation of A375 cells, while deletion of ZNF750 promoted the proliferative ability of A2058 cells (Figs. 2C-D).

\section{ZNF750 inhibits the migration, invasion and EMT markers' expression in melanoma cells}

As indicated in Figures 3A and 3B, transwell assay suggested that upregulation of ZNF750 significantly inhibited the migration and invasion of A375 cells, whereas ZNF750 knockdown obviously promoted the migration and invasion of A2058 cells. To investigate the functional effects of ZNF750 on the expression of EMT-associated markers in melanoma cells, we examined their expression in A375 and A2058 cells by western blot assay. Notably, overexpression of ZNF750 led to the upregulation of E-cadherin level, and downregulation of N-cadherin in A375 cells compared to control group. However, knockdown of ZNF750 caused the opposite effects in A2058 cells (Fig. 3C). Taken together, these data revealed the critical role of ZNF750 in regulating melanoma cell migration, invasion, and EMT.

\section{ZNF750 regulates melanoma cell growth via Wnt/3-catenin pathway}

Overexpression of ZNF750 obviously down-regulated the protein level of $\beta$-catenin in A375 cells as compared to the control group (Fig. 4A). The western blot analysis revealed that ZNF750 overexpression caused the notable down-regulation of cyclin D1, c-Myc and $\mathrm{Bcl}-2$. Besides, the protein levels of its downstream targets, MMP2 and MMP9, two proteases closely related to cell invasion and migration, were also strikingly decreased by ZNF750 upregulation (Fig. 4A). Consistently, knockdown of ZNF750 promoted the activation of $\mathrm{Wnt} / \beta$-catenin signaling and its downstream proteins in A2058 cells (Fig. 4B).

\section{Discussion}

In recent decades, the morbidity and mortality of malignant melanoma have increased significantly, with a high degree of malignancy [14]. It has been reported that uveal melanoma showed similar prognosis for Caucasian, Hispanic, Asian, and African American [15]. The specific etiology of melanoma is not clear, and ultraviolet light (UV) exposure $[16,17]$ is believed 


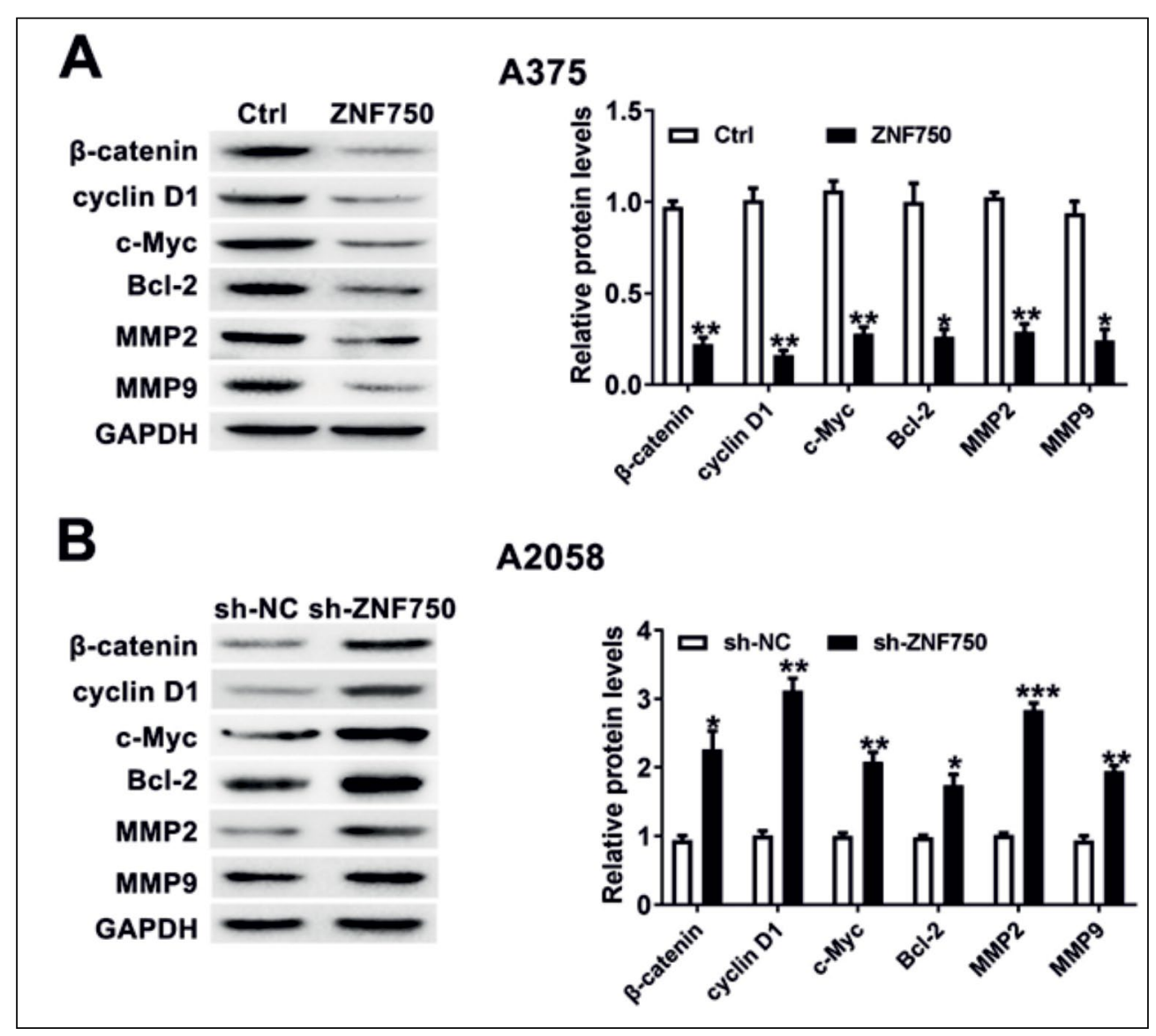

Figure 4. ZNF750 regulates melanoma progression via Wnt/ $\beta$-catenin pathway. A-B. Western blot assay was performed to detect the expression of $\beta$-catenin, cyclin D1, c-Myc, Bcl-2, MMP2 and MMP9 in A375 (A) and A2058 (B) cells. Results are presented as mean $\pm \mathrm{SD}$. ${ }^{*} \mathrm{p}<0.05,{ }^{*} \mathrm{p}<0.01,{ }^{* * *} \mathrm{p}<0.001$

to be a main cause. Although some patients can be cured by surgical removal of the tumor tissue, patients with metastatic disease and have poor sensitivity to radiotherapy and chemotherapy are accompanied by poor prognosis and high mortality $[4,18]$. Therefore, it is of great theoretical and clinical significance to identify the promising therapeutic molecules or targets and explore the molecular mechanisms underlying melanoma progression, thus to improve the prognosis of melanoma. Importantly, we found the downregulation of ZNF750 in human melanoma tissues and cells. According to the clinical-pathological analysis, ZNF750 expression was closely correlated with tumor size, lymph node metastasis and Clark classification in patients with melanoma. In patients with metastatic disease, those with melanotic melanomas exhibited significantly shorter overall survival and disease-free survival than those with amelanotic lesions, thus, inhibition of melanogenesis appears a rational adjuvant approach to the therapy of metastatic melanoma [19]. The present study indicated that the overall survival rate of high ZNF750 expression was higher than that of low ZNF750 expression, indicating that low ZNF750 expression predicted poor prognosis. Therefore, the results in this study suggested that ZNF750 may be a new potential therapeutic target for the treatment of melanoma. In human uveal tract and ocular melanoma, CYP27B1 and CYP24A1 were highly expressed in melanoma cells [20], and further study indicated that CYP24A1 expression was inversely correlated with melanoma progression [21]. The expression of ROR $\alpha$ and ROR $\gamma$ was also lower in melanomas than in nevi and decreased during melanoma progression, indicating their potential roles [22].

Previous studies have demonstrated the involvement of ZNF750 in cancer progression and metastasis in various cancer types. In different types of squamous cell carcinoma, ZNF750 is specifically mutated, deleted, and poorly expressed, and could drive epidermal differentiation and inhibit cell growth and migration through regulating lncRNA TINCR [23]. In oral squamous cell carcinoma, ZNF750 inhibited 
cell migration, invasion, and decreased the expression of matrix metalloproteinase 28, cyclin B1 and mesenchymal marker neural cadherin [24], and also have been reported to inhibit the malignant progression by regulating tumor vascular microenvironment [9]. In addition, in esophageal squamous cell carcinoma, low expression of ZNF750 was demonstrated to be closely related to the resistance to chemoradiotherapy, positive lymph node metastasis, and poor prognosis $[11,25]$, and downregulation of ZNF750 increased cell growth, migration, and invasion [8]. In other non-squamous cell carcinomas such as nasopharyngeal carcinomas, ZNF750 has also been shown to have an inhibitory effect on malignant tumor progression [7]. Analogously, these findings inspired us to explore whether ZNF750 exerts a crucial role in melanoma progression. As expected, ZNF750 elevation antagonized cell proliferation, migration, invasion, and EMT, which were aggravated after ZNF750 knockdown. These data suggested that ZNF750 might act as a pivotal participator in the progression of melanoma.

Cumulative evidence has suggested that the Wnt/ $/ \beta$-catenin pathway plays multiple roles in regulating various physiological processes in diverse human cancers $[26,27]$. Increasing evidence has suggested that abnormal activation of $\mathrm{Wnt} / \beta$-catenin pathway is necessary for the initiation and progression of melanoma [28-30]. Wnt $/ \beta$-catenin signaling has been reported to play an important role in regulating melanoma cellular processes such as cell growth, apoptosis, invasion, EMT and immunity [31-33]. It seems that the blockage of activation in the $\mathrm{Wnt} / \beta$-catenin signaling can inhibit tumor development, thus raise hopes for the discovery of new anticancer drug targets $[34,35]$. It raises the possibility that $\mathrm{Wnt} / \beta$-catenin signaling may be involved in the suppressive role of ZNF750 in the progression of melanoma. To verify it, we analyzed the expression levels of $\beta$-catenin, a core component of $\mathrm{Wnt} / \beta$-catenin signaling pathway. As expected, ZNF750 significantly inhibited the expression of $\beta$-catenin. It is known that cyclinD1, c-Myc, Bcl-2, MMP2 and MMP9 are common downstream molecules of $\mathrm{Wnt} / \beta$-catenin signaling pathway, which have been proved to be closely related to cell proliferation, apoptosis and metastasis [17, 27]. ZNF750 has significant inhibitory effects on the expression of these molecules, indicating that ZNF750 hinders the activation of $\mathrm{Wnt} / \beta$-catenin pathway. Together, these results suggest that the inhibitory role of ZNF750 in melanoma cell proliferation and invasion may through the Wnt/-catenin signaling pathway. Notably, these findings could provide a better understanding on the pathogenesis of melanoma and may contribute to the diagnosis and treatment of the disease.
In summary, this is the first evidence demonstrating the obvious low expression of ZNF750 in human melanoma tissues and cells. Importantly, ZNF750 expression was correlated with clinical characteristics including tumor size, lymph node metastasis, and Clark classification. The present study also proved that ZNF750 inhibited the proliferation, migration, invasion and EMT of melanoma cells in vitro by blocking the activation of $\mathrm{Wnt} / \beta$-catenin signaling. However, the role of ZNF in animal models of melanoma remains to be further studied. Hence, these findings contribute to better understanding on the regulatory role of ZNF750 in the pathogenesis of melanoma and provide promising treatments for anti-melanoma therapies.

\section{Competing interests}

The authors state that there are no conflicts of interest to disclose.

\section{Availability of data and materials}

All data generated or analyzed during this study are included in this published article.

\section{Authors' contributions}

QT conceived and designed the experiments, YD and GZL analyzed and interpreted the results of the experiments, JL and JJL performed the experiments

\section{References}

1. Howlader N, Noone A, Krapcho M et al. SEER stat fact sheets: Melanoma of the skin. National Cancer Institute SEER Database 2011.

2. Thiery JP, Sleeman JP. Complex networks orchestrate epithelial-mesenchymal transitions. Nat Rev Mol Cell Biol. 2006; 7(2): 131-142, doi: 10.1038/nrm1835, indexed in Pubmed: 16493418 .

3. Nakamura M, Tokura Y. Epithelial-mesenchymal transition in the skin. J Dermatol Sci. 2011; 61(1): 7-13, doi: 10.1016/j. jdermsci.2010.11.015, indexed in Pubmed: 21167690.

4. Afzal MZ, Mercado RR, Shirai K. Efficacy of metformin in combination with immune checkpoint inhibitors (anti-PD-1/ anti-CTLA-4) in metastatic malignant melanoma. J Immunother Cancer. 2018; 6(1): 64, doi: 10.1186/s40425-018-0375-1, indexed in Pubmed: 29966520.

5. Sen GL, Boxer LD, Webster DE, et al. ZNF750 is a p63 target gene that induces KLF4 to drive terminal epidermal differentiation. Dev Cell. 2012; 22(3): 669-677, doi: 10.1016/j. devcel.2011.12.001, indexed in Pubmed: 22364861.

6. Cassandri M, Smirnov A, Novelli F, et al. Zinc-finger proteins in health and disease. Cell Death Discov. 2017; 3: 17071, doi: 10.1038/cddiscovery.2017.71, indexed in Pubmed: 29152378.

7. Zhang P, He Q, Lei Y, et al. mA-mediated ZNF750 repression facilitates nasopharyngeal carcinoma progression. Cell Death Dis. 2018; 9(12): 1169, doi: 10.1038/s41419-018-1224-3, indexed in Pubmed: 30518868. 
8. Otsuka R, Akutsu Y, Sakata H, et al. ZNF750 expression is a potential prognostic biomarker in esophageal squamous cell carcinoma. Oncology. 2018; 94(3): 142-148, doi: 10.1159/000484932, indexed in Pubmed: 29216641.

9. Pan Li, Yang H, Xu C, et al. ZNF750 inhibited the malignant progression of oral squamous cell carcinoma by regulating tumor vascular microenvironment. Biomed Pharmacother. 2018; 105: 566-572, doi: 10.1016/j.biopha.2018.06.001, indexed in Pubmed: 29890464.

10. Choi S, Yu V, Ko JY, et al. Abstract 174: Functional characterization ofZNF750in esophageal squamous cell carcinoma. Tumor Biology. 2019, doi: 10.1158/1538-7445.am2019-174.

11. Otsuka R, Akutsu Y, Sakata H, et al. ZNF750 expression as a novel candidate biomarker of chemoradiosensitivity in esophageal squamous cell carcinoma. Oncology. 2017; 93(3): 197-203, doi: 10.1159/000476068, indexed in Pubmed: 28558382.

12. Liu W, Peng Y, Tobin DJ. A new 12-gene diagnostic biomarker signature of melanoma revealed by integrated microarray analysis. PeerJ. 2013; 1: e49, doi: 10.7717/peerj.49, indexed in Pubmed: 23638386.

13. Kim HY, Lee H, Kim SH, et al. Discovery of potential biomarkers in human melanoma cells with different metastatic potential by metabolic and lipidomic profiling. Sci Rep. 2017; 7(1): 8864, doi: 10.1038/s41598-017-08433-9, indexed in $\mathrm{Pu}-$ bmed: 28821754.

14. Johansson M, Brodersen J, Gøtzsche PC, et al. Screening for reducing morbidity and mortality in malignant melanoma. Cochrane Database Syst Rev. 2019; 6: CD012352, doi: 10.1002/14651858.CD012352.pub2, indexed in Pubmed: 31157404.

15. Shields CL, Kaliki S, Cohen MN, et al. Prognosis of uveal melanoma based on race in 8100 patients: The 2015 Doyne Lecture. Eye (Lond). 2015; 29(8): 1027-1035, doi: 10.1038/ eye.2015.51, indexed in Pubmed: 26248525.

16. Craig S, Earnshaw $\mathrm{CH}$, Virós A. Ultraviolet light and melanoma. J Pathol. 2018; 244(5): 578-585, doi: 10.1002/path.5039, indexed in Pubmed: 29380860.

17. Ko J, Geller A, Swetter S. Melanoma. Am Can Society's Oncol Pract. 2018: 485-501, doi: 10.1002/9781118592168.ch34.

18. Grossman D, Altieri D. Drug resistance in melanoma: mechanisms, apoptosis, and new potential therapeutic targets. Cancer Metastasis Rev. 2001; 20(1-2): 3-11, doi: 10.1023/a:1013123532723, indexed in Pubmed: 11831644.

19. Brożyna AA, Jóźwicki W, Carlson JA, et al. Melanogenesis affects overall and disease-free survival in patients with stage III and IV melanoma. Hum Pathol. 2013; 44(10): 2071-2074, doi: 10.1016/j.humpath.2013.02.022, indexed in Pubmed: 23791398.

20. Markiewicz A, Brożyna AA, Podgórska E, et al. Vitamin D receptors (VDR), hydroxylases CYP27B1 and CYP24A1 and retinoid-related orphan receptors (ROR) level in human uveal tract and ocular melanoma with different melanization levels. Sci Rep. 2019; 9(1): 9142, doi: 10.1038/s41598-01945161-8, indexed in Pubmed: 31235702.

21. Brożyna AA, Jochymski C, Janjetovic Z, et al. CYP24A1 expression inversely correlates with melanoma progression: clinic-pathological studies. Int J Mol Sci. 2014; 15(10): 19000-19017, doi: 10.3390/ijms151019000, indexed in $\mathrm{Pu}-$ bmed: 25334067.
22. Brożyna AA, Jóźwicki W, Skobowiat C, et al. ROR $\alpha$ and ROR $\gamma$ expression inversely correlates with human melanoma progression. Oncotarget. 2016; 7(39): 63261-63282, doi: 10.18632/oncotarget.11211, indexed in Pubmed: 27542227.

23. Hazawa M, Lin DC, Handral H, et al. ZNF750 is a lineage-specific tumour suppressor in squamous cell carcinoma. Oncogene. 2017; 36(16): 2243-2254, doi: 10.1038/ onc.2016.377, indexed in Pubmed: 27819679.

24. Yang H, Pan Li, Xu C, et al. Overexpression of tumor suppressor gene ZNF750 inhibits oral squamous cell carcinoma metastasis. Oncol Lett. 2017; 14(5): 5591-5596, doi: 10.3892/ ol.2017.6908, indexed in Pubmed: 29113187.

25. Nambara S, Masuda T, Tobo T, et al. Clinical significance of gene expression, a novel tumor suppressor gene, in esophageal squamous cell carcinoma. Oncol Lett. 2017; 14(2): 1795-1801, doi: 10.3892/ol.2017.6341, indexed in Pubmed: 28789412.

26. Monga SP. Role of $\mathrm{Wnt} / \beta$-catenin signaling in liver metabolism and cancer. Int J Biochem Cell Biol. 2011; 43(7): 1021-1029, doi: 10.1016/j.biocel.2009.09.001, indexed in Pubmed: 19747566.

27. White BD, Chien AJ, Dawson DW. Dysregulation of $\mathrm{Wnt} / \beta$-catenin signaling in gastrointestinal cancers. Gastroenterology. 2012; 142(2): 219-232, doi: 10.1053/j.gastro.2011.12.001, indexed in Pubmed: 22155636.

28. Xue G, Romano E, Massi D, et al. Wnt $/ \beta$-catenin signaling in melanoma: Preclinical rationale and novel therapeutic insights. Cancer Treat Rev. 2016; 49: 1-12, doi: 10.1016/j. ctrv.2016.06.009, indexed in Pubmed: 27395773.

29. Kovacs D, Migliano E, Muscardin L, et al. The role of Wn$\mathrm{t} / \beta$-catenin signaling pathway in melanoma epithelial-to-mesenchymal-like switching: evidences from patients-derived cell lines. Oncotarget. 2016; 7(28): 43295-43314, doi: 10.18632/ oncotarget.9232, indexed in Pubmed: 27175588.

30. Brown K, Yang P, Salvador D, et al. WNT/ $\beta$-catenin signaling regulates mitochondrial activity to alter the oncogenic potential of melanoma in a PTEN-dependent manner. Oncogene. 2017; 36(22): 3119-3136, doi: 10.1038/onc.2016.450, indexed in Pubmed: 28092677.

31. Yaguchi T, Goto Y, Kido K, et al. Immune suppression and resistance mediated by constitutive activation of Wnt $/ \beta$-catenin signaling in human melanoma cells. J Immunol. 2012; 189(5): 2110-2117, doi: 10.4049/jimmunol.1102282, indexed in Pubmed: 22815287.

32. Sinnberg T, Levesque MP, Krochmann J, et al. Wnt-signaling enhances neural crest migration of melanoma cells and induces an invasive phenotype. Mol Cancer. 2018; 17(1): 59, doi: 10.1186/s12943-018-0773-5, indexed in Pubmed: 29454361.

33. Gurzu S, Beleaua MA, Jung I. The role of tumor microenvironment in development and progression of malignant melanomas-a systematic review. Rom J Morphol Embryol. 2018; 1: 23-8, indexed in Pubmed: 29940608.

34. Dihlmann S, von Knebel Doeberitz M. Wnt/beta-catenin-pathway as a molecular target for future anti-cancer therapeutics. Int J Cancer. 2005; 113(4): 515-524, doi: 10.1002/ ijc.20609, indexed in Pubmed: 15472907.

35. Yao H, Ashihara E, Maekawa T. Targeting the Wnt $/ \beta$-catenin signaling pathway in human cancers. Expert Opin Ther Targets. 2011; 15(7): 873-887, doi: 10.1517/14728222.2011.577418, indexed in Pubmed: 21486121.

Submitted: 14 April, 2020

Accepted after reviews: 27 October, 2020

Available as AoP: 13 November, 2020 